\title{
Rectal Cancer: Time for Precision Medicine?
}

\author{
Martin R. Weiser, MD \\ Memorial Sloan Kettering Cancer Center, New York, NY
}

Thirty years ago, the National Surgical Adjuvant Breast and Bowel Project (NSABP) RO1 study was published: a landmark randomized trial demonstrating a rather remarkable reduction in local recurrence from 25 to $16 \%$ when pelvic radiation was added to the treatment regimen of rectal cancer. ${ }^{1}$ These findings ushered in the modern era of multimodality therapy for rectal cancer including chemotherapy, radiation and surgery. Current local recurrence rates following combined modality therapy for rectal cancer are now routinely reported to be less than $10 \%$. In spite of the impressive results with multimodality therapy, our colleagues in Heidelberg argue in this issue of Annals of Surgical Oncology that radiation, one of the components of multimodality therapy, can be safely eliminated in a significant proportion of locally advanced (American Joint Committee on Cancer T3/4 or N1/2) rectal cancer patients. Is this sensible? What is the rationale for deviation from chemotherapy, radiation and surgery in the treatment of advanced rectal cancer patients?

Let us take a step back, and review the justification for the current multimodality treatment of patients with stage II (T3/T4N0) and stage III (TanyN1/N2) rectal cancer. The current standard includes chemoradiation, total mesorectal excision (TME), and adjuvant systemic chemotherapy. The primary goal of pelvic radiation is to prevent local recurrence and, in some cases, convert a patient from requiring abdominoperineal resection to a sphincter-sparing low anterior resection. The primary rationale for systemic chemotherapy is to reduce the risk of distant recurrence.

This editorial refers to the article available at doi:10.1245/s10434015-4832-5.

(C) Society of Surgical Oncology 2015

First Received: 6 August 2015;

Published Online: 1 September 2015

M. R. Weiser, MD

e-mail: weiser1@mskcc.org
With multiple publications demonstrating excellent oncologic outcomes, single-digit local recurrence rates, and a decreasing rate of abdominoperineal resection, trimodality therapy has gained widespread acceptance in the United States and many parts of the world. ${ }^{1-6}$

Why, then, should this standard be challenged? Critics have argued that stage II and stage III rectal cancer should not be treated as homogeneous groups, as there is growing evidence that not all such patients are high-risk. In addition, some are at higher risk for distant, rather than local, recurrence. Thus, treating all stage II and III rectal cancer patients with chemotherapy, radiation and surgery exposes many to unnecessary treatment-related toxicity and delays systemic chemotherapy in those at highest risk for distant relapse.

Gunderson and colleagues have shown that the risk of local recurrence in patients with rectal cancer can be stratified by $\mathrm{T}$ and $\mathrm{N}$ stage using an aggregation of data from several phase III North American rectal cancer adjuvant trials (NCCTG 794751, NCCTG 864751, and U.S. GI Intergroup 0114). ${ }^{7} \mathrm{TN}$ stage was categorized according to risk of recurrence: low (T1/2N0), intermediate (T1/2N1, T3N0), moderately high (T1/2N2, T3N1, T4N0), and high (T3N2, T4N1/2). Patients with intermediate-risk tumors had better outcomes than patients with moderately high and high-risk tumors, including lower rates of local recurrence (6-8 \% vs. $8-15 \%$ and $15-22 \%$, respectively) and improved overall survival (74-81\% vs. $61-69 \%$ and $33-48 \%)^{7}$ These data suggest that routine adjuvant radiation therapy may not be required for intermediate-risk tumors $(\mathrm{T} 1 / 2 \mathrm{~N} 1$ or $\mathrm{T} 3 \mathrm{~N} 0)$ when TME with negative radial margins has been performed. The final analysis of the Intergroup 0114 trial reported similar findings. ${ }^{8}$ A second pooled analysis attempted to dissect the effect of treatment on outcome. ${ }^{9}$ Data from five phase III North American rectal cancer trials conducted over a 13-year period (NSABP R01, NSABP R02, NCCTG 794751, NCCTG 864751, and U.S. GI Intergroup 0114) were aggregated, 
and the subsequent analysis compared outcomes of 3,791 patients according to $\mathrm{TN}$ stage and various treatment regimens: surgery alone (179 patients), surgery and radiation (281 patients), surgery and chemoradiotherapy (2,799 patients), or surgery and chemotherapy (532 patients). In the setting of intermediate-risk tumors (T1/2N1 and T3N0), no additional benefit in disease-free survival or overall survival was observed when radiation was added to chemotherapy after surgery. These analyses demonstrate heterogeneity in the risk of local recurrence, and provide a rationale for individualizing treatment.

Even in the setting of modern rectal cancer staging, there is a risk of overtreatment based on overstaging. This has been demonstrated by the German CAO/ARO/AIO 94 trial, which compared preoperative to postoperative longcourse chemoradiotherapy for clinical stage II and III rectal cancer. ${ }^{10}$ The preoperative treatment group demonstrated a statistically significant decrease in local recurrence rates after 5 years, without a difference in rates of distant metastasis. Additional benefits of preoperative versus postoperative chemoradiation included a significant decrease in short-term and long-term toxicity. The incidence of Grade 3 or higher toxicity was reduced from $40 \%$ in the postoperative group to $27 \%$ in the preoperative group $(p=0.001)$. In this study, overtreatment and errors in clinical staging could be assessed since one cohort went directly to surgery. In total, $18 \%$ of patients in the postoperative chemoradiation group that went immediately to surgery had pathologic stage I disease, indicating a limitation in preoperative imaging and overtreatment for a significant proportion of patients.

Another rationale for reassessing reflexive neoadjuvant therapy is improved surgery. Anatomic, or mesorectal, excision is now widely adopted. With removal of the rectum together with its cylindrical mesentery and associated nodal tissue by precise and sharp dissection between the visceral and parietal layers of the endopelvic fascia, total mesorectal excision ensures complete removal of all locoregional lymph nodes, while maintaining negative circumferential radial margin, minimizing blood loss, and preserving the autonomic pelvic nerves. Although no prospective randomized trial has compared total mesorectal excision with conventional proctectomy, numerous retrospective and cohort studies demonstrate excellent local recurrence-free survival in patients undergoing proper rectal cancer surgery. ${ }^{11-13}$

Early rectal cancer trials demonstrating improvement with radiation were performed before total mesorectal excision became the standard. ${ }^{14,15}$ The Dutch trial, published in 2001, was the first study to integrate standard surgery into a control arm, and underscored the importance of high-quality surgical technique. This trial compared the combination of preoperative radiation therapy and total mesorectal excision to mesorectal excision alone, demonstrating a significant $(p<0.001)$ decrease in local recurrence in patients receiving preoperative short-course radiation. Critics have argued that, although the quality of surgery was controlled, up to one-quarter of patients had inadequate mesorectal excision on pathologic analysis, resulting in a significantly worse outcome $(p<0.02){ }^{16}$ Long-term follow-up demonstrated that certain patient subgroups-those with node involvement, negative CRMS, and/or low-lying tumors (within $5-10 \mathrm{~cm}$ of the anal verge)-obtained the greatest benefit from preoperative radiation. ${ }^{17,18}$ These findings suggested that some subgroups treated with multimodality therapy may derive less benefit from the addition of chemoradiation. Furthermore, neoadjuvant radiation did not improve overall survival.

The short- and long-term toxicity of pelvic radiation may be the most compelling reason to reconsider reflexive neoadjuvant chemoradiation and to move toward a more individualized approach. In the United States, radiation is given daily over a period of 5 to 6 weeks. This treatment can be difficult to tolerate, and compliance is frequently poor ${ }^{18}$; debilitating short-term toxicities have been reported in up to $50 \%$ of patients, ${ }^{17}$ with up to $70 \%$ of patients unable to complete the entire course of treatment. ${ }^{18}$ Additionally, the long-term effects of radiation can lead to disabling complications. Radiation-related fibrosis and autonomic nerve injury are associated with increased fecal incontinence, urgency, and frequency. Higher rates of sexual and genitourinary dysfunction, as well as small bowel obstruction requiring surgical intervention, have also been observed. Compared to patients who do not receive chemoradiation, quality of life is poorer. ${ }^{18-22}$ Finally, chemoradiation may impair bone marrow reserve, which complicates the ability to deliver systemic chemotherapy in the metastatic setting.

In this issue of Annals of Surgical Oncology, Kulu and colleagues from Heidelberg compare cohorts of stage II and III rectal cancer patients treated with total mesorectal excision with or without neoadjuvant therapy. The retrospective review of a multicenter database includes patients with tumors less than $12 \mathrm{~cm}$ from the anal verge, treated between 2002 and 2013; radiation was utilized at the discretion of the treating surgeon for patients thought to be at high risk for positive margin, or for those requiring neoadjuvant therapy aimed at downsizing distal tumors for sphincter preservation. Of 454 patients, $342(75 \%)$ were radiated and $112(25 \%)$ were not radiated. Of the 342 who received neoadjuvant treatment, $130(38 \%)$ underwent chemoradiation and $212(62 \%)$ had short-course radiotherapy. After surgery, $32 \%$ of the irradiated patients and $46 \%$ of the surgery-only patients received adjuvant chemotherapy. Survival, recurrence, positive circumferential radial margin, and complications were not different in 
FIG. 1 Outline of the PROSPECT trial. This large-scale multi-institutional phase II/III randomized prospective trial compares neoadjuvant FOLFOX with selective use of chemoradiation. PROSPECT Preoperative Radiation Or Selective Preoperative Radiation and Evaluation before Chemotherapy and Total Mesorectal Excision (TME)

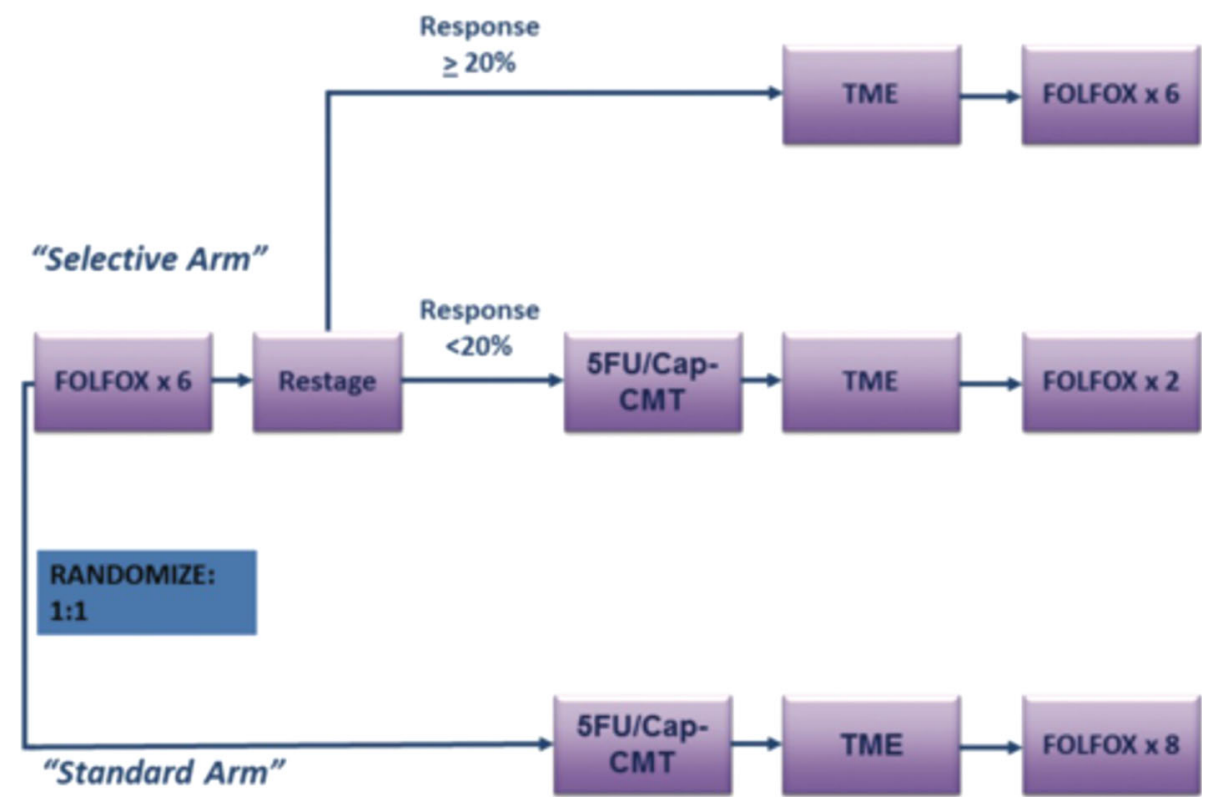

the irradiated and non-irradiated patients. Local recurrence rates were $3.8 \%$ in the irradiated patients and $4.5 \%$ in the non-irradiated patients. What can we conclude from these results? Most obvious is that the treating physicians involved in the study were expert clinicians and surgeons, able to successfully identify lower risk patients based on clinical examination and imaging. Can these results be generalized to a broader group of clinicians?

Currently there is an open prospective randomized trial seeking to determine whether preoperative radiation can be omitted in select patients. The PROSPECT trial, Preoperative Radiation Or Selective Preoperative Radiation and Evaluation before Chemotherapy and Total Mesorectal Excision (TME), is a large-scale, multi-institutional, phase II/III randomized prospective trial comparing neoadjuvant FOLFOX with selective use of chemoradiation (NCCTGN1048, NCT01515787), and is outlined in Figure 1. ${ }^{15,23}$ This trial is based on favorable data obtained in a pilot trial, in which locally advanced rectal cancer patients were initially treated with FOLFOX-based chemotherapy, without planned radiation therapy. The study cohort included $30 \%$ stage II and $70 \%$ stage III mid to low rectal cancers. Of the 30 patients receiving chemotherapy alone, all had complete R0 resection and eight $(27 \%)$ achieved a pathologic complete response, with no viable tumor detected in the resection specimen. ${ }^{24}$ All three patients who suffered recurrence had pulmonary metastasis, but no locally recurrent disease was observed.

In prospective randomized trial design PROSPECT is a multicenter trial which investigates whether standard combined modality treatment (CMT: 5-FU or capecitabinebased chemoradiation) can be replaced with selective use of neoadjuvant FOLFOX. The study randomly and equally assigns patients with $\mathrm{T} 1 / 2 \mathrm{~N} 1, \mathrm{~T} 3 \mathrm{~N} 0$, and $\mathrm{T} 3 \mathrm{~N} 1$ rectal cancer with tumors that are amenable to sphincter-preserving procedures, and without at-risk radial CRM, to either the "standard" or "selective" arm. Treatment in the standard arm consists of preoperative 5-FU or capecitabine-based chemoradiation, followed by TME and eight cycles of FOLFOX. Patients assigned to the selection arm receive six cycles of neoadjuvant FOLFOX and are then restaged. If clinical response to chemotherapy is observed in $20 \%$ or more, the patients immediately proceed to surgical resection and adjuvant FOLFOX (6 cycles); however, if tumor response is less than $20 \%$, the patients receive conventional combined-modality therapy before surgical resection and adjuvant FOLFOX (2 additional cycles). Patients in the selective arm who are found to have positive circumferential radial margins on final pathology undergo chemoradiation because of the increased risk for local recurrence. This study will determine whether neoadjuvant FOLFOX with selective use of chemoradiation can be safely used as an alternative to standard chemoradiation, without compromising the ability to perform a margin-negative surgical resection, achieve local control, and improve overall disease-free survival.

In summary, there has been substantial progress in rectal cancer therapy in the past decade. Although some patients still do not receive beneficial adjuvant and neoadjuvant treatments, at the same time the neoadjuvant paradigm means that others receive too much treatment. As Kulu and colleagues are alluding to, the field is ready for precision medicine, with tailoring of treatment to individual patient risk. Current trials, including PROSPECT, are attempting to meet these challenges and develop individualized treatment based on personal risk, thus maximizing 
oncologic outcome and quality of life while limiting treatment-related toxicity.

\section{REFERENCES}

1. [No authors listed]. NIH consensus conference. Adjuvant therapy for patients with colon and rectal cancer. JAMA. 264:1444-50, 1990

2. [No authors listed]. Prolongation of the disease-free interval in surgically treated rectal carcinoma. Gastrointestinal tumor study group. N Engl J Med. 312:1465-72, 1985

3. Fisher B, Wolmark N, Rockette H, Redmond C, Deutsch M, Wickerham DL, Fisher ER, Caplan R, Jones J, Lerner H, et al.: Postoperative adjuvant chemotherapy or radiation therapy for rectal cancer: results from NSABP protocol R-01. J Natl Cancer Inst. 80:21-9, 1988

4. Krook JE, O'Connell MJ, Wieand HS, Beart RW Jr, Leigh JE, Kugler JW, Foley JF, Pfeifle DM, Twito DI. A prospective, randomized evaluation of intensive-course 5-fluorouracil plus doxorubicin as surgical adjuvant chemotherapy for resected gastric cancer. Cancer 67:2454-8, 1991

5. Tepper JE, O'Connell MJ, Petroni GR, Hollis D, Cooke E, Benson AB III, Cummings B, Gunderson LL, Macdonald JS, Martenson JA. Adjuvant postoperative fluorouracil-modulated chemotherapy combined with pelvic radiation therapy for rectal cancer: initial results of intergroup 0114. J Clin Oncol. 15:2030-9, 1987

6. Wolmark N, Rockette H, Fisher B, Wickerham DL, Redmond C, Fisher ER, Jones J, Mamounas EP, Ore L, Petrelli NJ, et al. The benefit of leucovorin-modulated fluorouracil as postoperative adjuvant therapy for primary colon cancer: results from national surgical adjuvant breast and bowel project protocol C-03. J Clin Oncol. 11:1879-87, 1993

7. Gunderson LL, Sargent DJ, Tepper JE, O’Connell MJ, Allmer C, Smalley SR, Martenson JA, Haller DG, Mayer RJ, Rich TA, Ajani JA, Macdonald JS, Goldberg RM. Impact of $\mathrm{T}$ and $\mathrm{N}$ substage on survival and disease relapse in adjuvant rectal cancer: a pooled analysis. Int J Radiat Oncol Biol Phys. 54:386-96, 2002

8. Tepper JE, O'Connell MJ, Niedzwiecki D, Hollis DR, Benson AB III, Cummings B, Gunderson LL, Macdonald JS, Martenson JA, Mayer RJ. Adjuvant therapy in rectal cancer: analysis of stage, sex, and local control-final report of intergroup 0114. $J$ Clin Oncol. 20:1744-50, 2002

9. Gunderson LL, Sargent DJ, Tepper JE, Wolmark N, O’Connell MJ, Begovic M, Allmer C, Colangelo L, Smalley SR, Haller DG, Martenson JA, Mayer RJ, Rich TA, Ajani JA, MacDonald JS, Willett CG, Goldberg RM. Impact of T and N stage and treatment on survival and relapse in adjuvant rectal cancer: a pooled analysis. J Clin Oncol. 22:1785-96, 2004

10. Sauer R, Becker H, Hohenberger W, Rödel C, Wittekind C, Fietkau R, Martus P, Tschmelitsch J, Hager E, Hess CF, Karstens $\mathrm{JH}$, Liersch T, Schmidberger H, Raab R. German rectal cancer study group: preoperative versus postoperative chemoradiotherapy for rectal cancer. $N$ Engl J Med. 351:1731-40, 2004

11. Bokey EL, Ojerskog B, Chapuis PH, Dent OF, Newland RC, Sinclair G. Local recurrence after curative excision of the rectum for cancer without adjuvant therapy: role of total anatomical dissection. Br J Surg. 86:1164-70, 1999

12. Heald RJ, Moran BJ, Ryall RD, Sexton R, MacFarlane JK.: Rectal cancer: the Basingstoke experience of total mesorectal excision, 1978-1997. Arch Surg. 133:894-9, 1998

13. Merchant NB, Guillem J, Paty PB, Enker WE, Minsky BD, Quan SH, Wong D, Cohen AM.: T3N0 rectal cancer: results following sharp mesorectal excision and no adjuvant therapy. J Gastrointest Surg. 3:642-7, 1999
14. Folkesson J, Birgisson H, Pahlman L, Cedermark B, Glimelius B, Gunnarsson U.: Swedish rectal cancer trial: long lasting benefits from radiotherapy on survival and local recurrence rate. J Clin Oncol. 23:5644-50, 2005

15. [No authors listed]: Chemotherapy alone or chemotherapy plus radiation therapy in treating patients with locally advanced rectal cancer undergoing surgery. Available at: http://www.cancer.gov/ about-cancer/treatment/clinical-trials/search/view?cdrid=715321 Clinical Trials.gov Identifier: NCCTG-N1048

16. Nagtegaal ID, van de Velde CJ, van der Worp E, Kapiteijn E, Quirke P, van Krieken JH. Cooperative clinical investigators of the Dutch colorectal cancer group: macroscopic evaluation of rectal cancer resection specimen: clinical significance of the pathologist in quality control. J Clin Oncol. 20:1729-34, 2002

17. Kapiteijn E, Marijnen CA, Nagtegaal ID, Putter H, Steup WH, Wiggers T, Rutten HJ, Pahlman L, Glimelius B, van Krieken JH, Leer JW, van de Velde CJ. Dutch colorectal cancer group: preoperative radiotherapy combined with total mesorectal excision for resectable rectal cancer. N Engl J Med. 345:638-46, 2001

18. Peeters KC, Marijnen CA, Nagtegaal ID, Kranenbarg EK, Putter H, Wiggers T, Rutten H, Pahlman L, Glimelius B, Leer JW, van de Velde CJ. Dutch colorectal cancer group: the TME trial after a median follow-up of 6 years: increased local control but no survival benefit in irradiated patients with resectable rectal carcinoma. Ann Surg. 246:693-701, 2007

19. Aschele C, Pinto C, Cordio S, Rosati G, Tagliagambe A, Artale S, Rosetti P, Lonardi S, Boni L, Cionini L; on behalf of STAR Network Investigators. Properative fluorouracil (FU)-based chemoradiation with and without weekly oxaliplatin in locally advanced rectal cancer: Pathologic response analysis of the Studio Terapia Adiuvante Retto (STAR)-01 randomized phase III trial. J Clin Oncol. 27:CRA4008, 2009

20. Cercek A Goodman KA, Hajj C, Weisberger E, Segal NH, ReidyLagunes DL, Stadler ZK, Wu AJ, Weiser MR, Paty PB, Guillem JG, Nash GM, Temple LK, Garcia-Aguilar J, Saltz LB. Neoadjuvant chemotherapy first, followed by chemoradiation and then surgery, in the management of locally advanced rectal cancer. $J$ Natl Compr Canc Netw. 12:513-9, 2014

21. Chau I, Brown G, Cunningham D, Tait D, Wotherspoon A, Norman AR, Tebbutt N, Hill M, Ross PJ, Massey A, Oates J. Neoadjuvant capecitabine and oxaliplatin followed by synchronous chemoradiation and total mesorectal excision in magnetic resonance imaging-defined poor-risk rectal cancer. $J$ Clin Oncol. 24:668-74, 2006

22. Roh MS, Yothers GA, O'Connell MJ, Beeart RW, Pitot HC, Shields AF, Parda DS, Sharif S, Allegra CJ, Petrelli NJ, Landry JC, Ryan DP, Arora A, Evans TL, Soori GS, Chu L, Landes RV, Mohiuddin M, Lopa S, Wolmark N. The impact of capecitabine and oxaliplatin in the preoperative multimodality treatment in patients with carcinoma of the rectum: NASBP R-04. J Clin Oncol. 29:3503, 2011

23. [No authors listed]. PROSPECT: chemotherapy alone or chemotherapy plus radiation therapy in treating patients with locally advanced rectal cancer undergoing surgery. Available at: https://clinicaltrials.gov/ct2/show/NCT01515787 ClinicalTrials.gov Identifier: NCT01515787

24. Schrag D, Weiser MR, Goodman KA, Gonen M, Hollywood E, Cercek A, Reidy-Lagunes DL, Gollub MJ, Shia J, Guillem JG, Temple LK, Paty PB, Saltz LB. Neoadjuvant chemotherapy without routine use of radiation therapy for patients with locally advanced rectal cancer: a pilot trial. J Clin Oncol. 32:513-8, 2014 